\title{
Colitis amebiana necrosante, presentación de un caso
}

\author{
Necrotizing amebian colitis, case report
}

Miguel Briceño-Santana', Nagheli Mariann Barajas Grijalva', Ana Laura Vargas-Guzmán³, Carlos Manterola,4 y Nayely García Méndez²,5

\author{
${ }^{1}$ Hospital Star Médica Mérida. Yucatán, México. \\ ${ }^{2}$ Programa de Doctorado en Ciencias Médicas, Universidad de La Frontera. Temuco, Chile.

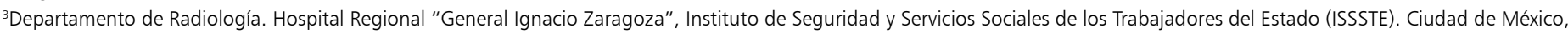 \\ México. \\ ${ }^{4}$ Centro de Excelencia en Estudios Morfológicos y Quirúrgicos (CEMyQ), Universidad de La Frontera. Temuco, Chile. \\ ${ }^{5}$ Hospital General del Centro Médico Nacional "La Raza" Instituto Mexicano del Seguro Social (IMSS). Ciudad de México, México.
}

Los autores declaran no tener conflictos de intereses.

No hubo apoyo financiero para la investigación, y publicación de este artículo.

Recibido: 13 de diciembre de 2019 / Aceptado: 17 de junio de 2020

\section{Resumen}

Introducción: La Organización Mundial de la Salud (OMS) y la Organización Panamericana de la Salud (OPS) han definido la amebiasis como la infección por Entamoeba histolytica, independientemente de los síntomas. La colitis amebiana necrosante es una forma rara de amebiasis que se asocia con una alta morbilidad y mortalidad. Caso clínico: Presentamos a un paciente femenino de 68 años, con antecedentes de diabetes mellitus tipo 2, con colitis amebiana necrosante con múltiples perforaciones, que fue sometida a una hemi-colectomía derecha con íleo-transverso anastomosis. Conclusiones: Se necesitan intervenciones efectivas para prevenir la colitis amebiana y terapias adicionales para tratar la colitis amebiana fulminante y mejorar los resultados.

Palabras clave: Entamoeba histolytica; disentería; colitis amebiana necrosante; enfermedades parasitarias; tinción ácida peryódico de Schiff.

\section{Introducción}

E ntamoeba histolytica es la tercera causa parasitaria de muerte más común en el mundo. Afecta a unos 500 millones de personas y mata a 75.000 de ellas anualmente. La prevalencia promedio mundial de amebiasis se ha reportado entre 8 y $10 \%{ }^{1}$.

Entamoeba histolytica es un potente patógeno, secreta proteinasas que disuelven los tejidos del hospedero, destruye las células del mismo al contacto y engloba a los eritrocitos; los trofozoitos de E. histolytica

\section{Abstract}

Background: Amebiasis has been defined by World Health Organization (WHO) and Pan American Health Organization (PAHO) as the infection with Entamoeba histolytica regardless of symptoms. Necrotizing amoebic colitis is a rare clinical form of amebosis that is associated with high morbidity and mortality. Case Report: We present a 68-years-old-female patient with necrotizing amoebic colitis with multiple perforations who survived after right hemicolectomy with ileus-transverse anastomosis. Conclusions: Effective interventions to prevent amebic colitis, and additional therapies to treat fulminant amebic colitis are needed to improve outcomes.

Key words: Entamoeba histolytica; dysentery, amoebic colitis; parasitic diseases; peryodic acid -Schiff reaction. invaden la mucosa intestinal causando la colitis amebiana (CA). Aunque no se conoce la prevalencia real de CA, las áreas que tienen altas tasas de infección amebiana incluyen: India, África, México, países de América Central y del Sur². La afectación del sigmoides es menos común y puede permanecer sin diagnóstico ${ }^{3}$. Sin embargo, en forma muy ocasional, la enfermedad toma un curso agudo fulminante; cuando se instala un cuadro abdominal de CA necrosante, se estima que la letalidad varía de 55 a 100\% si el diagnóstico y el tratamiento se retrasan. La falta de consulta médica, tratamiento y/o sospecha de 
un diagnóstico precoz permiten que la $\mathrm{CA}$ se presente como un abdomen agudo y el diagnóstico será confirmado tardíamente por anatomía patológica. La CA puede co-existir y confundirse con la enfermedad inflamatoria intestinal (EII) agravándose su pronóstico cuando se dan tratamientos a base de corticosteroides para EII y no se prescribe tratamiento antiamebiano ${ }^{4}$.

Presentamos un paciente con CA necrosante con múltiples perforaciones, que sobrevivió después de una hemicolectomía derecha con anastomosis íleo-transversal.

Este reporte fue escrito siguiendo las pautas del informe de caso CARE (Consensus-based clinical case report guideline development $)^{5}$.

\section{Caso clínico}

\section{Antecedentes}

Paciente de 68 años, originaria de Mérida, Yucatán, México. Ingresó a urgencias del Hospital Star Médica, Mérida. Portadora de diabetes mellitus tipo 2 (DM2) en tratamiento con metformina. Alérgica al paracetamol, dextropropoxifeno, sulfas y alcohol.

\section{Historia actual}

Refería dolor abdominal de moderada intensidad, progresivo, de dos meses de evolución, acompañado de distensión abdominal, diarrea crónica, en ocasiones deposiciones disentéricas, fiebre no cuantificada, así como malestar general y artralgias. En una primera atención médica, a la exploración física estaba afebril y presentaba dolor abdominal en el hipocondrio derecho. En esa ocasión fue manejada con analgésicos y dada de alta.

\section{Evolución}

Dos semanas más tarde, continuó con dolor en el hipocondrio derecho y el cuadrante inferior derecho del abdomen, siendo interpretada como una infección del tracto urinario y prescribiéndose una quinolona. En el quinto día de tratamiento, ella continuó con empeoramiento de la sintomatología, por lo que se realizó una ecografía abdominal que reportó asas intestinales dilatadas, indicándosele esta vez $500 \mathrm{mg}$ de nitazoxanida durante tres días por sospecha de una parasitosis. Más adelante tuvo una caída desde su cama, golpeándose la región lumbar y la cadera derecha, por la que fue ingresada al Departamento de Urgencias del mismo hospital. En el examen físico general se consignó Glasgow 15, presión arterial de 100/60 $\mathrm{mmHg}$, temperatura $37^{\circ} \mathrm{C}$ y obesidad GII. Se describió a la paciente en posición anti-álgica y refería aumento del dolor en el hipocondrio derecho; se detectó una masa abdominal palpable en la fosa ilíaca derecha. Una radiografía de la columna lumbar detectó una espondilolistesis L4-L5 y desgarro parcial del músculo ilíaco derecho. Fue evaluada por neurocirujano quien mencionó un probable compromiso radicular solicitando una resonancia magnética que fue informada como un proceso inflamatorio en ambos psoas, compatibles con probables hematomas y/o abscesos. La radiografía abdominal simple mostró un asa fija en el flanco derecho, con edema inter-asas y niveles hidro-aéreos (Figura 1). Se realizó una tomografía computarizada de abdomen, observándose engrosamiento de la pared y neumatosis en el colon ascendente, asociado a líquido libre, con índice de atenuación correspondiente a líquido no puro en la fosa iliaca y corredera parieto-cólica derecha, compatible con abscesos (Figuras 2 y 3 ).

\section{Pruebas de laboratorio}

Hemoglobina $11,3 \mathrm{~g} / \mathrm{dl}$, hematocrito $31,8 \%$, volumen corpuscular medio $88,6 \mathrm{fL}$, recuento de leucocitos 21.450 céls $/ \mathrm{mm}^{3}$ (neutrófilos $85 \%$ ), plaquetas $145.000 / \mathrm{mm}^{3}$. Proteína C reactiva 376,23 mg/l. Glucosa $243 \mathrm{mg} / \mathrm{dl}$, urea $112,2 \mathrm{mg} / \mathrm{dl}$, creatininemia $1,27 \mathrm{mg} / \mathrm{dl}$, sodio $131 \mathrm{mmol} / \mathrm{l}$, potasio $4,5 \mathrm{mmol} / 1$, cloro $95 \mathrm{mmol} / 1$, bilirrubinemia total $0,26 \mathrm{mg} / \mathrm{dl}$, procalcitonina 9,69 $\mathrm{ng} / \mathrm{ml}$ (parámetro 2-10 ng/ $\mathrm{ml}$ ) alto grado de progresión a infección sistémica grave. Estudio de TORCH negativo. Se decidió su ingreso a la Unidad de Cuidados Intensivos.

\section{Intervención quirúrgica}

Previo consentimiento informado de acuerdo con la NOM-004-SSA3-2012, se realizó una laparotomía exploradora bajo anestesia general balanceada. Se describieron los hallazgos de un líquido hemo-purulento en

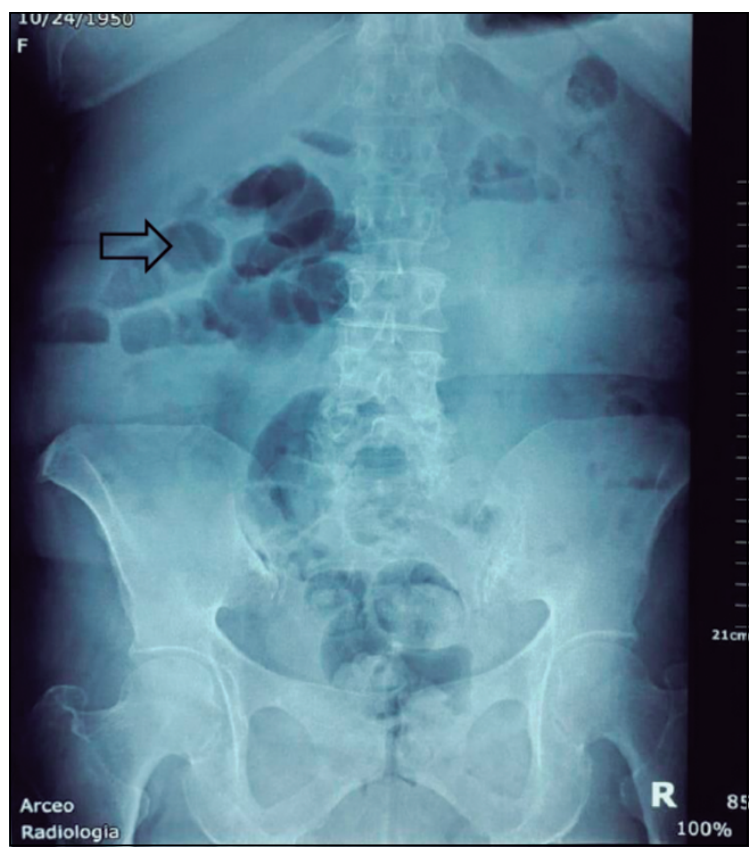

Figura 1. Radiografía simple de abdomen. Se observa asa intestinal fija en el flanco derecho, con edema inter-asas y niveles hidroaéreos (flecha). 


\section{Caso Clínico}

necrótica, lográndose identificar trofozoitos de ameba,

la cavidad abdominal, epiplón mayor envuelto hacia la fosa ilíaca derecha, hemi-colon derecho con gangrena predominantemente en el ciego, por lo que se decidió realizar una hemi-colectomía derecha con anastomosis íleo-transversal. El examen histopatológico del colon resecado mostró áreas ulceradas del ciego con superficie con tinción peryódica con ácido-Schiff (PAS). En la pared apendicular se identificó una tumoración bien definida con células fusiformes, compatible con un tumor del estroma gastrointestinal (GIST), con bajo potencial maligno; de tamaño 1,5 cm x 1,00 mm (Figura 4).
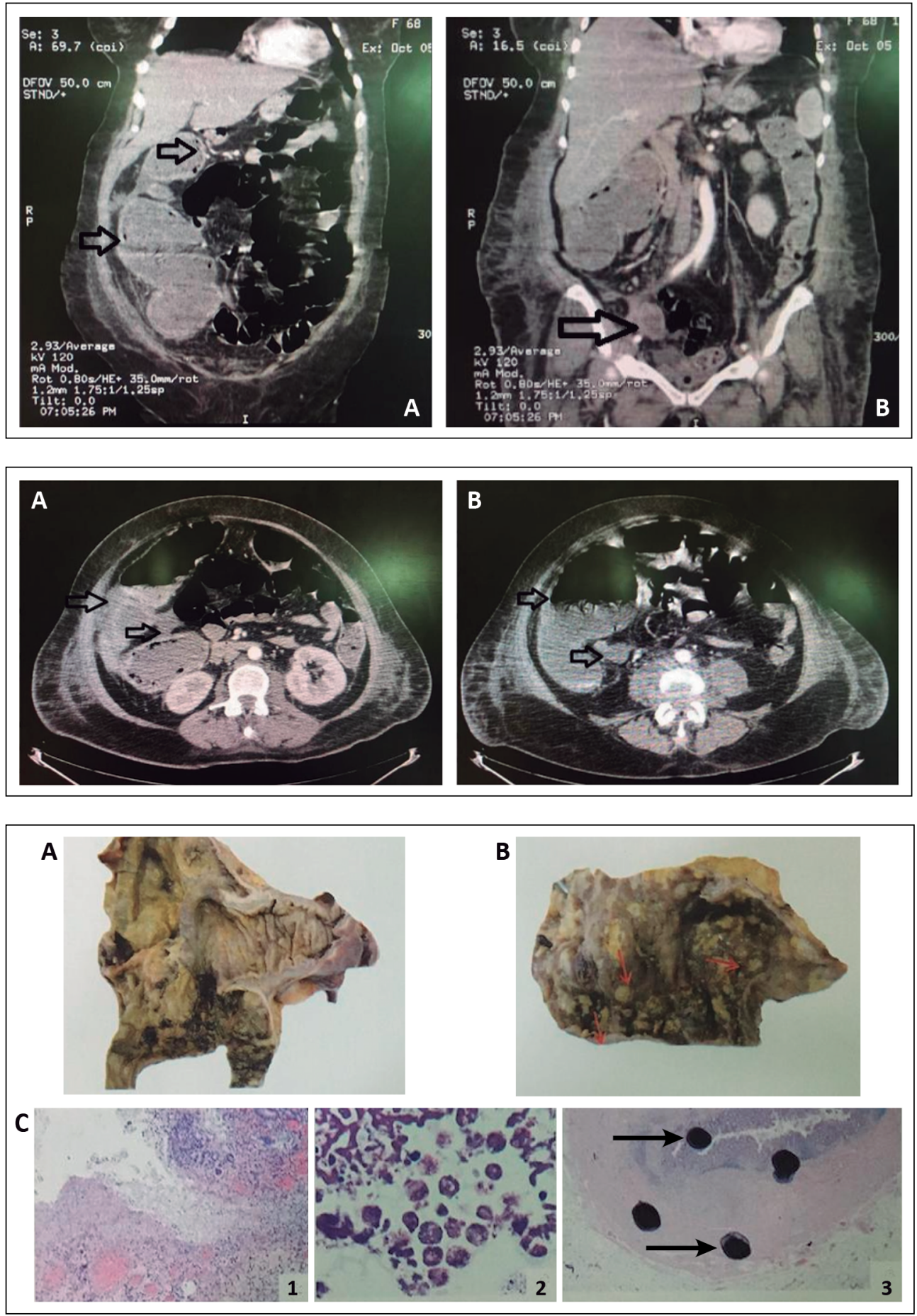

Figura 2. Tomografía computarizada de abdomen contrastada, con reconstrucción coronal. A. Distensión y neumatosis en el grosor de la pared del ciego y el colon ascendente, asociado con cambios por edema de la grasa pericólica. B. Se identifica el engrosamiento del trayecto apendicular sugerente de un proceso inflamatorio.

Figura 3. Tomografía computarizada de abdomen, cortes axiales. A. Engrosamiento de la pared y neumatosis en el colon ascendente, asociada a líquido libre, con índice de atenuación correspondiente a líquido no puro en la fosa ilíaca y corredera parieto-cólica derecha, compatible con abscesos. B. Nivel hidro-aéreo en el colon derecho asociado con líquido libre en su borde mesentérico.

Figura 4. Pieza quirúrgica. A. Íleon, válvula ileocecal y ciego, esta última con pared adelgazada y necrótica con mucosa que muestra ulceración extensa. B. Corte de íleon ascendente, la presencia de úlceras transversales recubiertas por tejido amarillento, de aspecto necrótico. C. Corte histológico de ciego. 1) Zonas ulceradas del ciego con superficie necrótica. 2) Se identificaron trofozoitos (flecha) de E. histolytica o ameba spp. (Lente ocular 40x), tinción peryódica ácido de Schiff. 3) Tumor bien definido con células fusiformes en la pared apendicular, compatible con un tumor del estroma gastrointestinal (GIST). 


\section{Resolución}

La paciente cursó con una mejoría gradual, se completaron esquemas de antimicrobianos por vía intravenosa después de la cirugía y egresó tras 16 días de hospitalización, en favorables condiciones.

\section{Discusión}

La enterocolitis necrosante, también conocida como colitis amebiana fulminante, en un alto porcentaje evoluciona a la muerte, a pesar de recibir tratamiento médico y quirúrgico, básicamente por evolucionar hacia una falla orgánica múltiple; en el caso de nuestra paciente, la evolución fue favorable con el uso de antimicrobianos, antiamebianos, más la cirugía y el apoyo de cuidados intensivos.

La colitis fulminante aguda secundaria a CA es una complicación rara de la amebiasis intestinal y tiende a complicarse con una perforación intestinal en aproximadamente menos de $1 \%$ de los casos, pero se asocia con una alta tasa de mortalidad, sobre $50 \%$. Los síntomas varían desde diarrea leve hasta disentería intensa (94-100\%). La fiebre y la pérdida de peso debido a la diarrea crónica están presentes en $40 \%$ de los pacientes 6 .

$\mathrm{La}$ amebiasis se adquiere por la ingesta de $E$. histolytica presente en alimentos y aguas contaminados con quistes maduros de los cuales se liberan trofozoitos en el lumen intestinal siendo ésta la forma patógena de la infección ${ }^{7,8}$.

Varias especies de protozoos del género Entamoeba colonizan a los humanos, pero no todas están asociadas con la enfermedad. Entamoeba histolytica es bien reconocida como una ameba patógena, asociada con infección intestinal y extra-intestinal ${ }^{7}$.

A menudo, la amebiasis, no es sospechada inicialmente. La EII se diagnostica con mayor frecuencia, debido al cuadro clínico en que los síntomas abdominales en la amebiasis se superponen con los síntomas de la EII. Aproximadamente $0,5 \%$ de los pacientes desarrollan disentería. La complejidad puede deberse también a que los pacientes con EII pueden tener amebiasis; principalmente en países desarrollados constituye un reto el diagnóstico diferencial. En ausencia de diarrea, el cirujano puede sospechar de una lesión en el cuadrante inferior derecho del abdomen que requiera tratamiento quirúrgico ${ }^{8}$.

Hay muchas especies del protozoo del género Entamoeba, que colonizan el tubo digestivo; en la literatura médica se reporta que ocho de ellas infectan a los humanos: E. histolytica, Entamoeba dispar, Entamoeba coli, Entamoeba moshkovskii, Entamoeba hartmanni, Entamoeba polecki, Entamoeba gingivalis y Entamoeba bangladeshi. Sin embargo, sólo la especie E. histolytica, ha sido reconocida mundialmente como causante patógeno de afecciones intestinales y extra-intestinales en el ser humano ${ }^{10}$.
La frecuencia de perforación de colon por amebiasis en México ha disminuido considerablemente; no obstante, en los últimos años se ha descrito un caso similar de colitis amebiana fulminante con neumatosis cecal, que requirió laparotomía y en quien se documentó la presencia de $E$. histolytica en la pared del colon ${ }^{11}$.

Dentro de los métodos diagnósticos utilizados (en muestras de heces frescas o fijadas, suero, material de abscesos amebianos hepáticos) se encuentran: la tinción de PAS, microscopia electrónica, detección de antígenos, reacción de polimerasa en cadena (RPC) convencional y RPC en tiempo real (en heces). Procedimientos con isoenzimas tienen la desventaja de tener diferentes niveles de sensibilidad y especificidad; sin embargo, esta técnica es capaz de identificar entre E. histolytica y E. dispar. La serología con determinación de anticuerpos anti $E$. histolytica se realiza mediante técnicas de ELISA y por hemaglutinación indirecta (IHA) siendo esta última más específica ${ }^{12}$.

El aprendizaje que compartimos en este caso clínico es que $10 \%$ de las personas infectadas por $E$. histolytica desarrollan síntomas, mientras que $90 \%$ tiene una colonización asintomática. Las amebas invasoras pueden desarrollar complicaciones, poseen la capacidad de adhesión, daño tisular y perforación intestinal $(1,9-9,1 \%)$ siendo esta última la más letal. Por lo que se debe generar mayor conciencia clínica y patológica de la amebiasis intestinal en los países desarrollados y que suele a menudo tratarse equivocadamente como una EII. Dentro del protocolo de estudio en los pacientes se recomienda un estudio de heces (antígeno fecal) o serología amebiana.

\section{Conclusiones}

La complicación más grave de la infección amebiana es la colitis amebiana necrosante, su perforación y el absceso hepático. La colitis fulminante por CA, a menudo no se sospecha clínicamente; incluso, en áreas endémicas se puede confundir clínica y endoscópicamente con EII, debido a que ambas entidades pueden co-existir y cuando no se sospecha amebiasis se puede agravar el pronóstico con el tratamiento efectuado, especialmente si se usan corticosteroides. En muchas ocasiones las EII enmascaran el cuadro. El diagnóstico requiere un alto índice de sospecha clínica por parte del equipo médico. Sin embargo, el diagnóstico de colitis fulminante de etiología amebiana rara vez se realiza antes de la intervención quirúrgica siendo el estudio histopatológico el que entrega la certeza diagnóstica.

La profilaxis mediante el manejo y consumo adecuado de alimentos y una higiene óptima, junto con el diagnóstico y tratamiento específico y oportuno con antiamebianos, ya sea tisulares y/o de acción en el lumen intestinal, son útiles en la prevención de complicaciones como la descrita en este caso. 


\section{Caso Clínico}

\section{Referencias bibliográficas}

1.- Ozdogan M, Baykal A, Aran O. Amebic perforation of the colon: Rare and frequently fatal complication. World J Surg 2004; 28 (9): 926-9. doi: 10.1007/s00268-004-7503-4.

2.- Stanley S L. Amoebiasis. Lancet 2003; 361(9362): 1025-34. doi: 10.1016/S01406736(03)12830-9.

3.- Goel A, Bansal R, Kaur N, Arora P. Isolated sigmoid colon perforation in an unsuspected case of amoebic colitis. Indian J Med Spec [Internet] 2015; 6(4): 168-9. Available from: http://dx.doi.org/10.1016/j.injms.2015.09.003.

4.- Gupta S S, Singh O, Shukla S, Raj M K. Acute fulminant necrotizing amoebic colitis: A rare and fatal complication of amoebiasis: a case report. Cases J 2009; 2(9): 1-4. doi: 10.4076/1757-1626-2-6557.

5.- Gagnier J J, Kienle G, Altman D G, Moher D, Sox H, Riley D, et al. The CARE guidelines: Consensus-based clinical case report guideline development. J Clin Epidemiol 2014; 67(1): 46-51. doi: 10.1016/j.jclinepi.2013.08.003.

6.- Dogra V K, Gupta D, Kashyap R, Nand L, Sondhi S. Intestinal amebiasis presenting as life threatening lower GI bleed-A rare presentation. Ann Trop Med Public Health 2017; 10 (1): 244 6. doi:10.4103/1755-6783.205554.

7.- Parasites-Amebiasis - Entamoeba histolytica infection. Pathogen \& Environment. Centers for Disease Control and Prevention. https://www. cdc.gov/parasites/amebiasis/pathogen.html.

8.- Shirley D A, Moonah S. Fulminant amebic colitis after corticosteroid therapy: a systematic review. PLoS Negl Trop Dis 2016; 10 (7): 1-13. https://doi.org/10.1371/journal.pntd.0004879.

9.- Athié-Gutiérrez C, Rodea-Rosas H, GuízarBermúdez C, Alcántara A, Montalvo-Javé E E. Evolution of surgical treatment of amebiasisassociated colon perforation. J Gastrointest Surg 2010; 14 (1): 82-7. doi 10.1007/s11605009-1036-y.

10.- Ali I K M. Intestinal amebae. Clin Lab Med [Internet] 2015; 35(2): 393-422. Available from: http://dx.doi.org/10.1016/j.cll.2015.02.009.

11.- Rodríguez-Wong U, Rodríguez-Medina U. Fulminant amoebic colitis with cecal pneumatosis. Rev Gastroenterol Mex 2018; 83(4): 453-4. doi:10.1016/j.rgmx.2018.02.007.

12.- Bhilkar K A, Chande C, Singh A. Amoebic peritonitis associated with cirrhosis of liver: a case report. Int J Curr Res Rev 2017; 9(18): 16-7. doi: 10.7324/IJCRR.2017.9183. 